\section{Arteriovenous malformations}

\section{DONNA WINDERBANK-SCOTT}

St Mary's Hospital, Paddington, London, UK

E: donna@gambyte.co.uk

\section{AVM Support UK}

\section{www.avmsupport.org.uk}

This site is designed as a patient information portal as well as a support forum and concentrates almost exclusively on cerebrovascular AVMs. The site is fairly basic but comprehensive, and the author and date of the last update are recorded on each page. The section on investigations and what to expect is particularly useful for an anxious patient; there are many personal experiences and testimonies to read. A facility for altering text size is useful for the visually impaired and the overall layout is logical and well organised. However, the site is mainly text-based, has few illustrations or pictures and is probably too basic for medical professionals. **

\section{Birthmarks}

\section{www.birthmarks.us}

This site has been written by a vascular malformations' specialist and contains many good, high-quality images including a variety of clinical photographs as well as CT scans, angiography images and $3-D$ reconstructions. The clinical information is broken down into the various types of malformation with separate pages for associated syndromes. Links to full-text PDF journal articles by various authors provide more in depth clinical information to a specialist level. There are Power-Point presentations to download, and several case studies. However, some links provide abstracts instead of full-text articles and there is no facility to enlarge the images. This site is very detailed and comprehensive; as such. it will probably be most useful for post-MRCS surgical trainees or when preparing presentations on a specific aspect of the field. ${ }^{* * *}$

\section{Emedicine.com}

\section{http://www.emedicine.com/plastic/topic468.htm}

From the student staple website, this article provides a brief overview of vascular malformations including history, diagnosis, pathophysiology and aetiology. The information is not particularly detailed and is probably more appropriate for medical students. There are only four images which are very poor quality and not enlargeable without a paid subscription fee. However, the 1-2 line summaries of vascular malformation syndromes are useful. **

\section{Vascular Disease Foundation}

\section{http://www.vdf.org/CVM/}

This American organisation's aim is to educate patients in aspects of vascular disease and risk-factor modification. The website covers all aspects of vascular disease with a section on congenital vascular malformations. The information in this section is patient-orientated but entirely text with no images. There are spelling mistakes and the content is difficult to read, with long, confusing prose. Several sections are still under development and are currently empty. For a large, multidisciplinary organisation, this site is disappointing.*

\section{NOVA - National Organisation of Vascular Anomalies http://www.novanews.org/}

Another American organisation website, which focuses on haemangiomas and birthmarks as well as AVM-related syndromes. There are plenty of photographs and further links to various research projects in this field. The clinical overviews are primarily aimed at patients, but are detailed enough for surgical trainees and contain full references. The site is fairly well laid out but has some formatting problems with text sizes and occasional missing characters. However, overall, the clinical information is very well written and comprehensive. ${ }^{* * *}$

\section{Personal digital assistant (PDA)-based drug formularies}

THOMAS HS FYSH

Department of Surgery, Royal Cornwall Hospital, UK

E: thomas.fysh@btinternet.com

The days of frantically searching for a dog-eared copy of the BNF could soon be over. If you own a PDA or smartphone, the chances are that all this information could be available at your fingertips in an instant. The PDA-based formularies are geared-up mainly for the US/Canadian market and this has practical implications when using them in the UK, specifically in the NHS (such as licensed preparations, trademarks and the use of non-SI units).

\section{$B N F$}

The obvious exception is the original BNF which is compiled by the BMA and Royal Pharmaceutical Society of Great Britain, available for Palm OS. It can be obtained on-line from $<$ www.pharmpress.com $>$ and is updated every 6 months but only comes on an SD card which must be ordered. It is not cheap at $£ 99$ per edition (every 6 months) + VAT for NHS users but has the distinct advantage of being the only formulary here which was designed for the UK. A big disappointment is that, despite pledges to make it available to pocket PC (PPC) since 2004, this has yet to happen. We are promised that it is in the pipeline as is a downloadable version. Unfortunately, there are no plans for a Symbian OS version so Nokia smartphone users won't be able to benefit. The software itself is reliable and easy to use, and the information is comprehensive, as you might expect. Its cost and lack of compatibility let it down. ${ }^{* * *}$

\section{Epocrates}

My personal favourite is Epocrates Rx which is available for free in its most basic (but perfectly adequate) form from 